\title{
Hormonal control of behavior: novel mechanisms and model organisms
}

\author{
Barney A. Schlinger ${ }^{1,2,3,4}$
}

Received: 16 October 2017 / Revised: 29 October 2017 / Accepted: 30 October 2017 / Published online: 3 November 2017

(C) Springer-Verlag GmbH Germany 2017

Endocrinology is traditionally defined as the study of chemical signals present in blood that act on target tissues at some distance from the tissue source of the signal. These chemical signals, or hormones, can produce innumerable effects on those target tissues, from effects that occur locally and almost instantly, to some that impact tissues massively and last a lifetime. That degree of influence is dictated by numerous factors starting with the time and amount of hormone released into the circulatory system and ending with the diverse cellular mechanisms through which the hormones influence cell and tissue function (Nelson 2015).

The early years of behavioral endocrine research placed a significant focus on the steroid hormones and on determining the amounts of hormone in blood. In some cases, wild vertebrates were the subjects, illustrated by classic summary works such as that of Farner and Wingfield (1980). Alongside these kinds of studies were others aimed at elucidating hormone-dependent intracellular signaling pathways, work largely utilizing laboratory or domesticated animal models (e.g., O’Malley 1984). Such studies also revealed the significant role for enzyme-catalyzed reactions that, by their

Barney A. Schlinger

schlinge@lifesci.ucla.edu

1 Department of Integrative Biology and Physiology, University of California, Los Angeles, 610 Charles Young Drive, Los Angeles, CA 90095, USA

2 Department of Ecology and Evolutionary Biology, University of California, Los Angeles, 610 Charles Young Drive, Los Angeles, CA 90095, USA

3 Laboratory of Neuroendocrinology, University of California, Los Angeles, 610 Charles Young Drive, Los Angeles, CA 90095, USA

4 Smithsonian Tropical Research Institute, Balboa, Panama City, Panama metabolism of hormones, provided significant control over cellular processes, including in the central nervous system (Callard et al. 1978; McEwen 1981). Foundational concepts like these have been refined and reshaped over the subsequent years to sharpen our understanding of the vast and diverse reach of hormones in the control of animal behavior. With the availability now of many animal genomes and sophisticated bioinformatics tools, we are experiencing a new wave of fundamental research into the ways in which hormones influence the cellular transcriptome to define those genes and gene networks on which hormones act to modify cellular function and behavior.

Where these approaches have become especially exciting is that we have come more and more to appreciate just how many individual tissues within a single organism can be targets of a particular hormone to influence the animal's behavioral phenotype. We also now appreciate that some signals that were once considered just hormones are seen to be essential neural-to-neural signals and thus not really hormones at all (Saldanha et al. 2011; Mitre et al. 2017). Some hormones seem to act on virtually every significant tissue in the body and perform a myriad of functions by a multiplicity of mechanisms and control of innumerable tissue-specific genes and gene networks. Altogether, these various hormonal actions serve to control many facets of behavior. While a focus on how and when hormones are released into the bloodstream remains a popular area of research, it is these latter mechanistic functions that are of current greatest interest, including on free-living animals.

While we are generally familiar with the clinical implications of advances in endocrine physiology, they are also of great interest to biologists asking diverse questions about the anatomical, physiological and behavioral capabilities of wild animals. Evolutionary biologists consider the ways in which endocrine systems participate in 
establishing adaptive phenotypes that help define ecological life history patterns and which may also become the substrate upon which natural and/or sexual selection act and through which species evolve (Ketterson et al. 2009; Hau and Goymann 2015).

It is these latter ideas that inspired the content of this special issue. And as the focus is on hormonal control of behavior, we will be considering these mechanisms largely as they apply to the central nervous system and to skeletal muscles, two systems that involve a great diversity of tissues and cells and circuits. But the spotlight captures other systems as well including the ways in which endocrine systems themselves are modified, how non-neuromuscular systems can be altered to impact behavior and the ways in which sensory systems can become captured to modify the neuromuscular output.

In as much as they have the capacity to reach virtually all of the tissues in the body, have marked impacts on behavior and are so popular for study (Adkins-Regan 2005), there is a particular focus in this collection of papers on steroidal hormones. As will hopefully be obvious, even with a focus on steroids that have been investigated for many years, there are still new discoveries that expand our concepts about the mechanisms of hormone action and the evolution of tissue hormone responsiveness.

This latter point is readily apparent in the paper by Whittaker et al., who provide evidence for a new steroiddependent communication signal. Using a species of songbird, an animal group typically thought to have relatively poor olfactory capabilities, the authors build on their prior work showing that not only can these birds utilize odors for intra-specific communication but the bird's uropygial gland, the gland that produces oils used for feather maintenance and water-proofing, has evolved expression of androgen receptors. Moreover, with the binding of androgen to these receptors, the release of volatiles used for olfactory communication can be adaptably modified to influence social interactions.

While the previous paper highlights the ways in which an endocrine signal influences the output of a novel (at least in a songbird) sensory signal, hormones can also capture sensory systems themselves to augment how a variety of communication signals can be received and perceived. Yue et al. describe evidence they have collected showing that the retina of goldfish is a target of sex steroids. In this particular case, by the binding of estradiol to one of the estrogen receptors, retinal responses to visual stimuli can be rapidly modulated in males. These effects might also influence the male's behavioral responses to the visual stimulus of females in a manner that promotes mating success. These results, while highlighting the retina as a sex-steroid target, also illustrates that sex steroids can have rapid cellular actions by their interactions with membrane receptors, in addition to their well-established actions regulating transcription via intracellular receptors.

In the previous paper, it is very likely that functional estradiol was formed in the retina from circulating androgen by the local expression of the estrogen synthetic enzyme aromatase. Whereas it is well-known that the aromatase enzyme is active and functional in the central nervous systems of many vertebrates (Balthazart and Ball 2013), males have largely been the focus in the past given that females are seen to make their bioactive estradiol in their ovaries. In the next paper, Cornil turns this traditional narrow focus on its head by highlighting the many ways in which central aromatization can have a profound impact on brain and behavior in female vertebrates.

Given it is the primary location for neural circuits controlling sensory perception, behavioral motivation and motor output, research into the mechanisms underlying hormonal control of behavior have traditionally focused on the brain. Behavioral output, however, is achieved by muscle contractions and skeletal muscles are also significant hormone targets. It is becoming increasingly clear that the evolution of steroid sensitivity can capture the use of distinct neuromuscular systems to promote unique behaviors (Nasipak and Kelley 2012). This concept is well-illustrated in the next several papers. Monks et al. provide an overview of how androgen receptor expression in skeletal muscle plays a key role in the sexual differentiation of neuromuscular systems, especially in mammalian model systems. Placed in the background of seminal work on Xenopus species, Mangiemele and Fuxjager extend these concepts in describing their recent work on the evolution of androgen receptor expression in hindlimb muscles that promote the unusual "foot-flagging" behavior of some species of frogs. Evidence for the evolution of unique androgen-dependent traits is also seen in reptiles with evidence provided by Johnson et al. for androgen receptor expression in forelimb muscles as a potential source of variation in locomotor and "push-up" displays by anolis lizards.

The degree of androgen receptor expression in skeletal muscles can also function as the basis for the development of very complex physical displays. The work of Fuxjager and Schlinger on small tropical birds called manakins provides support for this hypothesis. Moreover, this work shows how investigation into the muscular transcriptome can enlighten perspectives on the hormonal control of behavior and the evolution of hormonal control systems involving peripheral targets.

The ways through which hormones influence behavior by altering transcription are more fully developed in the final two papers. Smith et al. present new data describing transcriptomes of the brain region controlling the electric organ discharge (EOD) in two species of knifefishes. The EOD, an electric communication signal, is under gonadal 
steroid control and the two species studied by Smith et al. exhibit a distinctive sexual dimorphism of their EODs. Their data not only identifies expression of genes encoding steroid signaling molecules, but also genes encoding a variety of ion channels and other neuronal properties that may underlie sex and species differences in electric communication.

The final paper by Frankl-Vilches and Gahr provides an up-to-date review that addresses the complex steroidal signaling underlying the well-characterized neural song system of oscine songbirds. This system has been the focus of intensive research on neuroplasticity, neural control of learning, sensory-motor integration and more, and steroids play fundamental roles in each of these processes (Schlinger and Brenowitz 2017). This new paper serves to highlight crossspecies variability in song learning and song structure based on variability in steroid-dependent transcriptional control. Diverse patterns of steroid receptor expression, the possible role of steroid-receptor splice variants, and the evolution of steroid-receptor binding sites on unique gene targets provide several examples of hormone action that could only be revealed by accurate genomic and transcriptomic analyses.

I hope that these papers make it apparent that new models of behavioral endocrinology are still waiting to be discovered, as are new insights into the mechanisms by which hormones exert their influence on behavior. The comparative approach is always worthwhile and with the surge in affordable sequencing capabilities and an emerging crop of biologically curious computational specialists, the future of comparative behavioral physiology is bright indeed.

\section{References}

Adkins-Regan E (2005) Hormones and animal social behavior. Princeton University Press, Princeton
Balthazart J, Ball GF (2013) Brain aromatase, estrogens and behavior. Oxford University Press, Oxford

Callard GV, Petro Z, Ryan KJ (1978) Phylogenetic distribution of aromatase and other androgen-converting enzymes in the central nervous system. Endocrinology 103:2283-2290

Farner DS, Wingfield JC (1980) Reproductive endocrinology of birds. Ann Rev Physiol 42:457-72

Hau M, Goymann W (2015) Endocrine mechanisms, behavioral phenotypes and plasticity: known relationships and open questions. Front Zool 12(Suppl 1):S7. https://doi. org/10.1186/1742-9994-12-S1-S7

Ketterson ED, Atwell JW, McGlothlin JW (2009) Phenotypic integration and independence: hormones, performance, and response to environmental change. Integ Comp Biol 49:365-379

McEwen BS (1981) Neural gonadal steroid actions. Science 211:1303-1311

Mitre M, Minder J, Morina EX, Chao MV, Froemke RC (2017) Oxytocin modulation of neural circuits. Curr Topics Behav Neurosci. https://doi.org/10.1007/7854_2017_7

Nasipak B, Kelley DB (2012) Developing laryngeal muscle of Xenopus laevis as a model system: Androgen-driven myogenesis controls fiber type transformation. Dev Neurobiol 664-675

Nelson RJ (2015) An introduction to behavioral endocrinology. Sinauer Assoc, Sunderland

O’Malley BW (1984) Steroid hormone action in eucaryotic cells. J Clin Invest 74:307-312

Saldanha CJ, Remage-Healey L, Schlinger BA (2011) Synaptocrine signaling: steroid synthesis and action at the synapse. Endocrine Revs 32:532-549

Schlinger BA, Brenowitz EA (2017) Neural and hormonal control of birdsong. In: Pfaff DW, Joëls M (eds) Hormones, brain, and behavior, vol 2, 3rd edn. Academic Press, Oxford, pp 255-290 